\title{
Effect of Acute Immobilization on the Expression Levels of Atrophy-Related Ubiquitin Ligase in Mice Fed a High-fat Diet
}

\author{
AYUMI GOTO*1), TAKAMASA TSUZUKI*2), TOSHINORI YOSHIHARA*3), \\ SHINYA FURUICHI*4), KEI TSUKIOKA*4), LEI ZHI*4), RYO KAKIGI*5), HISASHI NAITO*4) \\ *1) Sportology Center, Juntendo University Graduate School of Medicine, Tokyo, Japan, *2) Faculty of Health and Sports \\ Science, Juntendo University, Chiba, Japan, ${ }^{* 3}$ COI project, Juntendo University, Tokyo, Japan, *4) Graduate School of \\ Health and Sports Science, Juntendo University, Chiba, Japan, *5) Department of Physiology, Juntendo University Faculty of \\ Medicine, Tokyo, Japan
}

\begin{abstract}
Introduction: Skeletal muscle has a large plasticity in response to changes in extracellular stimuli. It has been well known that prolonged periods of muscle disuse due to chronic bed rest or immobilization lead to skeletal muscle atrophy and that the maintenance of muscle mass is one of key factors for Quality of Life (QOL). Recently, it has been reported that high-fat diet-obesity accelerates by 14 days denervation-induced muscle atrophy and protein degradation in mice. Furthermore, we have previously showed that immobilization for 3 day increased muscle RING finger 1 (MuRF1) and atrogin-1/muscle atrophy F-box (MAFbx) in high-fat diet-obesity compared with normal diet (unpublished data). It is notable that the amount of muscle mass decreased a few days after immobilization, but little is known about the acute change in the expression level of atrophy-related ubiquitin ligases (MuRF1 and atrogin-1/MAFbx) before an decrease in muscle mass in obesity mouse skeletal muscle. The purpose of this study was to investigate the short-term effect of immobilization on the expression levels of MuRF1 and atrogin-1/MAFbx in mice fed a high-fat diet.
\end{abstract}

Methods: This study was approved by the Juntendo University Animal Care Committee (H28- 14). Fourteen male C57BL/6J mice (6 weeks old) were randomly divided into two groups: 1) normal diet (ND, $\mathrm{n}=7), 2$ ) high-fat diet (HFD, $\mathrm{n}=7$ ). HFD were fed a high-fat diet ( $60 \%$ calories from fat) for 16 weeks, while ND received a chow diet (10\% calories from fat). Following 16 weeks of each experimental diet intake, all mice were lightly anaesthetized with the inhalant isoflurane and attached the casting material. One hindlimb was immobilized ( $\mathrm{Imm}$ ) in the plantar flexion position with casting tape, non-immobilized leg served as an internal control (Con). After $6 \mathrm{~h}$, animals were sacrificed and the soleus and plantaris muscles of both hindlimbs were removed and frozen in liquid nitrogen and stored at $-80^{\circ} \mathrm{C}$ for real-time RT-PCR. Statistical significance was analyzed by using two-way (diet and treatment) analysis of variance (ANOVA). Statistical significant level was set at $\mathrm{p}<0.05$. Results: Body weight and epididymal fat pad mass were significantly higher in the HFD compared with ND ( $<<$ 0.05). The absolute soleus and plantaris muscle weight were significantly higher in the HFD compared with ND. On the other hand, the relative soleus and plantaris muscle weight to body weight were significantly lower in the HFD compared with ND. However, these parameters were not altered during immobilization in HFD and ND. The expression level of atrogin-1/MAFbx mRNA in soleus muscle was significantly lower during immobilization in HFD and ND, whereas there was no alteration in plataris. On the other hand, the expression level of MuRF1 mRNA in soleus and plantaris muscle were no changes during immobilization in HFD and ND.

Conclusion: Short-term immoblization for $6 \mathrm{~h}$ did not affect the expression levels of atrophyrelated ubiquitin ligase during immobilization in mice fed a high-fat diet.

Corresponding author: Ayumi Goto

Sportology Center, Juntendo University Graduate School of Medicine

2-1-1 Hongo, Bunkyo-ku, Tokyo 113-8421, Japan

TEL: +81-3-3813-3111 E-mail: a-goto@juntendo.ac.jp

〔Received Dec. 19, 2017〕〔Accepted Feb. 13, 2018〕

Copyright (C) 2018 The Juntendo Medical Society. This is an open access article distributed under the terms of Creative Commons Attribution License (CC BY), which permits unrestricted use, distribution, and reproduction in any medium, provided the original source is properly credited. doi: $10.14789 /$ jmj. 2018.64.JMJ18-P46 
This study was supported in part by President's Grant for Interfaculty Collaboration, Juntendo University (A. Goto), Japan Society for the Promotion of Science Research Activity Start-up Grant number 16H07182 (A. Goto), the Descente Foundation for the Promotion of Sports Science (A. Goto).

Key words: skeletal muscle, atrophy, immobilization, obesity, mice 